\title{
Chirped Probe Pulse Femtosecond Coherent Anti-Stokes Raman Scattering Thermometry at 5 kHz in a Gas Turbine Model Combustor
}

Claresta N. Dennis ${ }^{1}$, Carson D. Slabaugh ${ }^{1}$, Isaac G. Boxx ${ }^{2}$, Wolfgang Meier ${ }^{2}$, Robert P. Lucht ${ }^{1}$

${ }^{1}$ School of Mechanical Engineering, Purdue University, 585 Purdue Mall, West Lafayette, Indiana 47906, USA

${ }^{2}$ German Aerospace Center (DLR), Institute of Combustion Technology, Pfaffenwaldring 38-40, 70569 Stuttgart, Germany

Corresponding Author: Claresta N. Dennis

Phone: 765-494-2708

Email: cfineman@purdue.edu

Colloquium: Diagnostics

Alternate: Turbulent Flames

Paper Length using Method \#1

\begin{tabular}{|l|c|}
\hline Main Text & 2668 \\
\hline References & 472 \\
\hline Figure 1 + Caption & 495 \\
\hline Figure 2 + Caption & 149 \\
\hline Figure 3 + Caption & 542 \\
\hline Figure 4 + Caption & 160 \\
\hline Figure 5 + Caption & 181 \\
\hline Figure 6 + Caption & 258 \\
\hline Figure 7 + Caption & 685 \\
\hline Figure 8 + Caption & 148 \\
\hline Figure 9 + Caption & 148 \\
\hline Total & $\mathbf{5 9 0 6}$ \\
\hline
\end{tabular}




\begin{abstract}
Single-laser-shot temperature measurements at $5 \mathrm{kHz}$ were performed in a model gas turbine combustor using femtosecond (fs) coherent anti-Stokes Raman scattering (CARS). The combustor was operated at a global equivalence ratio of 0.65 and $10 \mathrm{~kW}$ thermal power. Measurements were performed at various locations within the flame in order to resolve the spatial flame structure and compare to previously published studies. Power spectral density analysis of the temperature measurements yielded the characteristic thermo-acoustic pulsation frequency previously reported at $308 \mathrm{~Hz}$. These results demonstrate the usefulness of fs-CARS for the investigation of highly turbulent combustion phenomena. The spatial resolution of the single-laser shot temperature measurements was approximately $600 \mu \mathrm{m}$, the precision was approximately $\pm 2 \%$, and the estimated accuracy was approximately $\pm 3 \%$. The dynamic range was sufficient for temperature measurements ranging from $300 \mathrm{~K}$ to $2400 \mathrm{~K}$, although some detector saturation was observed for low temperature spectra.
\end{abstract}

\title{
Keywords
}

laser diagnostics; coherent anti-Stokes Raman scattering; ultrafast spectroscopy; gas turbine combustion; turbulent swirl flame

\section{Introduction}

Coherent anti-Stokes Raman scattering (CARS) spectroscopy has been applied extensively for gas phase combustion diagnostics. Many literature reviews and textbooks describe CARS theory in extensive detail, describing experimental and theoretical spectral modeling techniques. A 2010 survey of applications of CARS spectroscopy for reacting flow diagnostics highlights the many potential 
advantages of fs-CARS over traditional ns- and ps-CARS techniques [1]. Solid-state femtosecond lasers operate at high repetition rates and offer excellent shot-to-shot spectral stability, nearly eliminating the shot-to-shot spectral fluctuations encountered with typical broadband dye lasers used for nanosecond CARS. In addition, the nearly Fourier-transform limited broadband femtosecond laser pulses excite many Raman transitions at once, creating a strong coherence in the sample medium [2,3]. This allows fs-CARS temperature measurements based on the frequency-spread dephasing rate after initial excitation of the Raman coherence of the molecule by the pump and Stokes beams. If one uses a chirped-probe-pulse (CPP), a method introduced by Lang et al. [4], the temporal decay of the Raman coherence can be mapped with linear and nonlinear contributions onto the frequency of the CARS signal allowing single-laser-shot measurements. Experiments have shown the CARS signal is nearly independent of molecular collisions after the initial excitation of the coherence and the initial decay rate of the Raman coherence in gas phase measurements depends only on temperature $[5,6]$. Focusing on the first few picoseconds after excitation will result in collision-free measurements for pressures up to 20 bar [3]. This simplifies significantly the theoretical modeling of fs-CARS spectra and improves accuracy by eliminating the need for Raman linewidth information [1].

Since the review by Roy et al. [1], studies have demonstrated the improved accuracy of fs-CARS thermometry of reacting flows. Richardson et al. demonstrated excellent precision of the fs-CARS technique for flame measurements, with standard deviations of $1-1.5 \%$ of the mean temperature over a wide range of operating conditions [7]. Measurements were performed in a driven non-premixed hydrogen-air flame with nitrogen coflow and a turbulent methane-air Bunsen burner flame. Bangar et al. reported CPP fs-CARS temperature measurements of a hydrogen-air jet diffusion flame at $5 \mathrm{kHz}$ [8]. They were able to resolve temperature fluctuations occurring due to Kelvin-Helmholtz instabilities and vortices present from the interaction of hot combustion gases and cold ambient air. Measured temperatures ranged from 400 to $2500 \mathrm{~K}$ with $2 \%$ standard deviation. 
CPP fs-CARS has previously been applied only in mildly turbulent flames $[7,8]$. The purpose of the present work is to demonstrate the application of $5 \mathrm{kHz}$ CPP fs-CARS thermometry to swirl stabilized model combustors with very high levels of turbulence. In collaboration with the German Aerospace Center (DLR), measurements were performed on the DLR Dual-Swirl Gas Turbine Model Combustor (GTMC). The GTMC is a research-scale swirl burner designed to study thermo-acoustic oscillatory effects on combustion chemistry for technically relevant operating conditions. The burner has been extensively characterized previously for various global equivalence ratios and thermal powers using planar laser-induced fluorescence (PLIF) of $\mathrm{OH}, \mathrm{CH}$ and $\mathrm{H}_{2} \mathrm{CO}$, laser Doppler anemometry (LDA), $\mathrm{OH}^{*}$ chemiluminescence imaging, Raman scattering, two-line OH PLIF thermometry, and stereoscopic particle image velocimetry (PIV) [9-16]. The presence of an inner and outer recirculation zone and a precessing vortex core (PVC) has been well documented. A study by Weigand et al. [14] showed that mixing of hot products from the recirculation zones with the fresh gas influences the heatrelease rate and the reacting flow-field, leading to induced periodic motions of the inner and outer recirculation zones. A more recent study was reported by Boxx et al. [16]. They obtained spatial and temporal frequency content from $5 \mathrm{kHz}$ simultaneous stereoscopic PIV and OH-PLIF measurements and showed that the reaction zone conforms to the passage of large scale vortex structures associated with the PVC present within the flow-field.

In this study, CPP fs-CARS measurements were successfully performed throughout the highly turbulent reacting flow field of the GTMC. The measurements revealed very different temporal structure at different points in the flow. Power spectral density analysis was performed on the resulting temperature measurements to compare with previous studies. The spatial resolution of the technique was measured and found to be much better than previously reported for ns-CARS experiments. The accuracy and precision of these results is estimated to be within 3\%. Beam steering effects were minimal and not significant to the point that single-shot spectra could not be analyzed; no loss of signal was experienced. 


\section{Experimental System}

\section{Femtosecond CARS Optical System}

For this experiment, the fundamental output of a $2 \mathrm{~mJ}, 5 \mathrm{kHz}, 50 \mathrm{fs}$ Ti:Sapphire regenerative amplifier (Legend Elite Duo, Coherent, Inc.) was used to pump an optical parametric amplifier (OPA) and also to provide the laser pulses for the CARS probe and Stokes beams. Beam splitters were used to direct $90 \%$ of the amplifier energy to pump the OPA, $6 \%$ through a dispersive rod (30 cm, SF-10) to produce the chirped probe beam, and the remaining $4 \%$ to be used directly as the Stokes beam. The spectral bandwidths of the pump, Stokes, and CPP beams full width at half-maximum (FWHM) were $264 \mathrm{~cm}^{-1}, 406 \mathrm{~cm}^{-1}$, and $422 \mathrm{~cm}^{-1}$, respectively. 


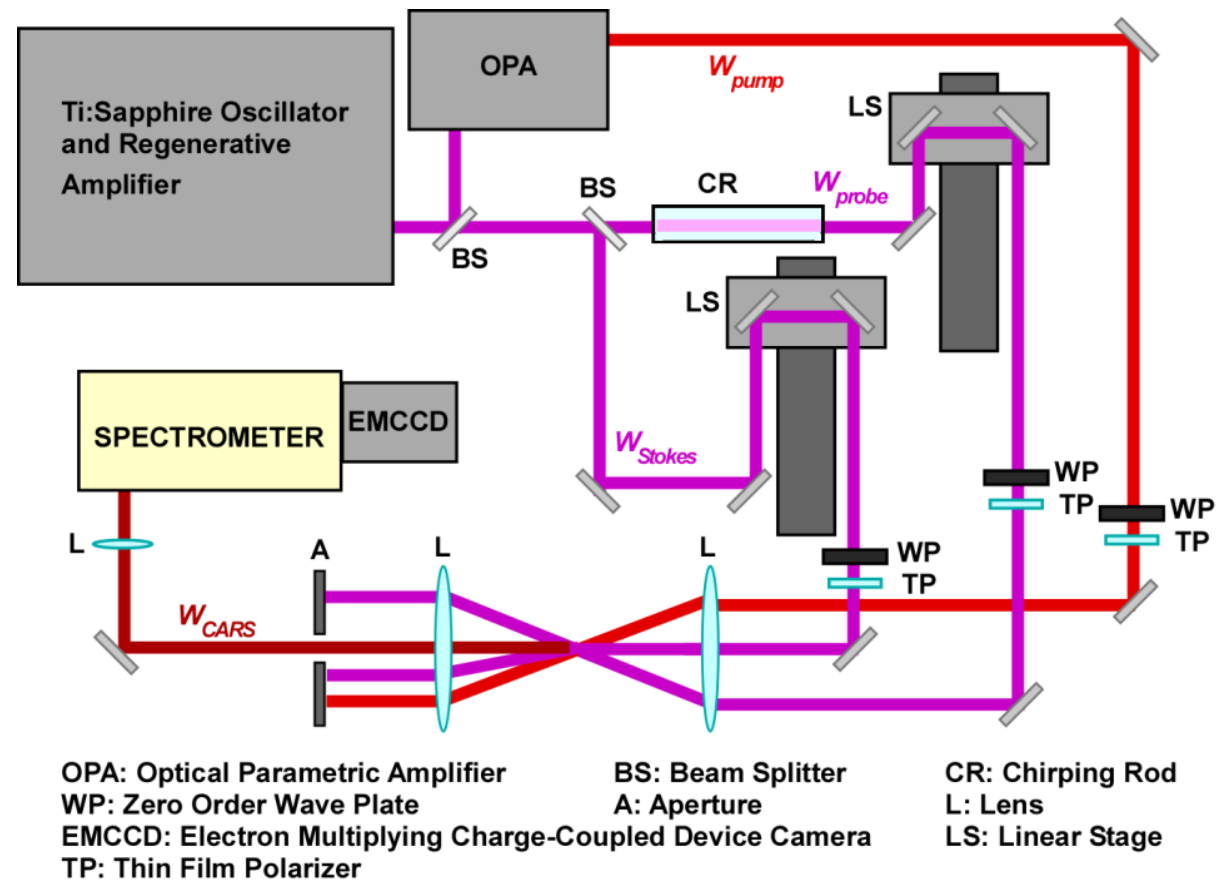

Figure 1: Schematic diagram of CPP fs-CARS experimental system.

For the CPP fs-CARS experiments, the spatial and temporal overlap among the pump, Stokes, and probe beams are critical. All three beams were focused to the probe volume using a 200-mm planoconvex lens in a phase-matched, folded BOXCARS geometry with a crossing angle of approximately $3^{\circ}$ [17]. At the probe volume, spatial overlap was achieved by focusing all three beams through a $50 \mu \mathrm{m}$ pinhole. Temporal overlap was confirmed by placing a $100 \mu \mathrm{m}$ thick Type-I BBO crystal at the probe volume and carefully adjusting the optical path lengths of the CPP and Stokes pulses using linear translation stages to maximize the intensity of sum-frequency signals generated from the combined beams.

The probe volume length was determined by translating the BBO crystal through the CARS probe volume along the propagation direction of the three laser beams. The nonresonant CARS signal generated inside the crystal was recorded and the CARS signal strength versus crystal position is shown in Figure 2. The probe volume length was approximately $400 \mu \mathrm{m}$, corresponding to the FWHM, or 600 
$\mu \mathrm{m}$ for the $10 \%$ to $10 \%$ of the maximum. The diameter of the probe volume was approximately $50 \mu \mathrm{m}$. The excellent spatial resolution of these measurements is probably due to the low beam divergence and excellent Gaussian mode quality of the ultrafast laser.

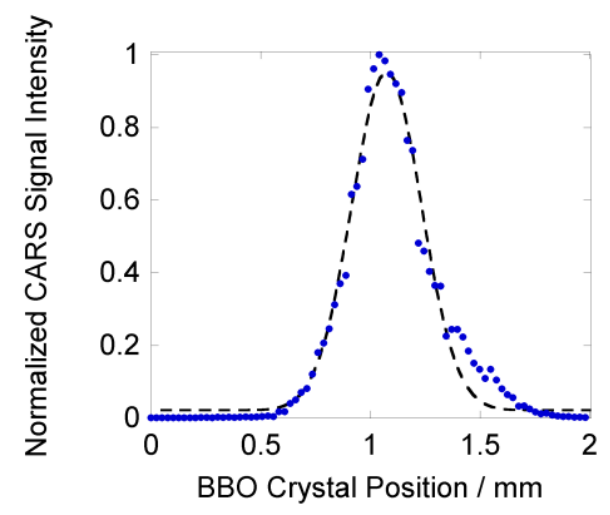

Figure 2: CARS probe volume length measurement.

The CPP fs-CARS signal beam, generated by the combination of spatially and temporally overlapped CPP, Stokes, and pump pulses, was then focused onto the entrance slit of a $0.25 \mathrm{~m}$ spectrometer. The CARS spectra were recorded using an electron multiplying charged coupled device (Andor iXon EMCCD) equipped with an optical mask to limit the signal illumination region to the bottom 50 rows of pixels. The CCD was vertically binned across the bottom 50 rows to allow for image acquisition at $5 \mathrm{kHz}$.

\section{Calibration Flame}

The 'Hencken burner' is a commonly used reference flame for combustion diagnostics [18]. The burner top is $37.5-\mathrm{mm}$-square and consists of a central burner plate with a fine honeycomb structure through which the oxidizer flows. Fuel is carried by hypodermic needles which protrude just above the 
honeycomb structure. Downstream of the burner face, the fuel and oxidizer flows rapidly mix and combust to produce a uniform flow of product gases.

For this experiment, the Hencken burner was used to produce stable, near adiabatic $\mathrm{H}_{2}$-air flames. Digital mass flow controllers were calibrated and used to control the volumetric flow rates of each of the gases. Hydrogen-air equivalence ratios of $0.3,0.5$, and 0.8 provided temperature standards at 1189, 1643, and $2168 \mathrm{~K}$ respectively. These standard temperatures were measured using the combined pure rotational/pure vibrational ns-CARS experiment developed by Satija and Lucht [19] and located in the same laboratory. The measured values are in agreement to within $0.2 \%$ of the calculated adiabatic flame temperature. The probe volume for the CPP fs-CARS measurements was located $35 \mathrm{~mm}$ above the center of the burner where the flame is most stable and product gases have reached equilibrium. Averaged experimental spectra and corresponding theoretical fits for each equivalence ratio are shown in Figure 3. 

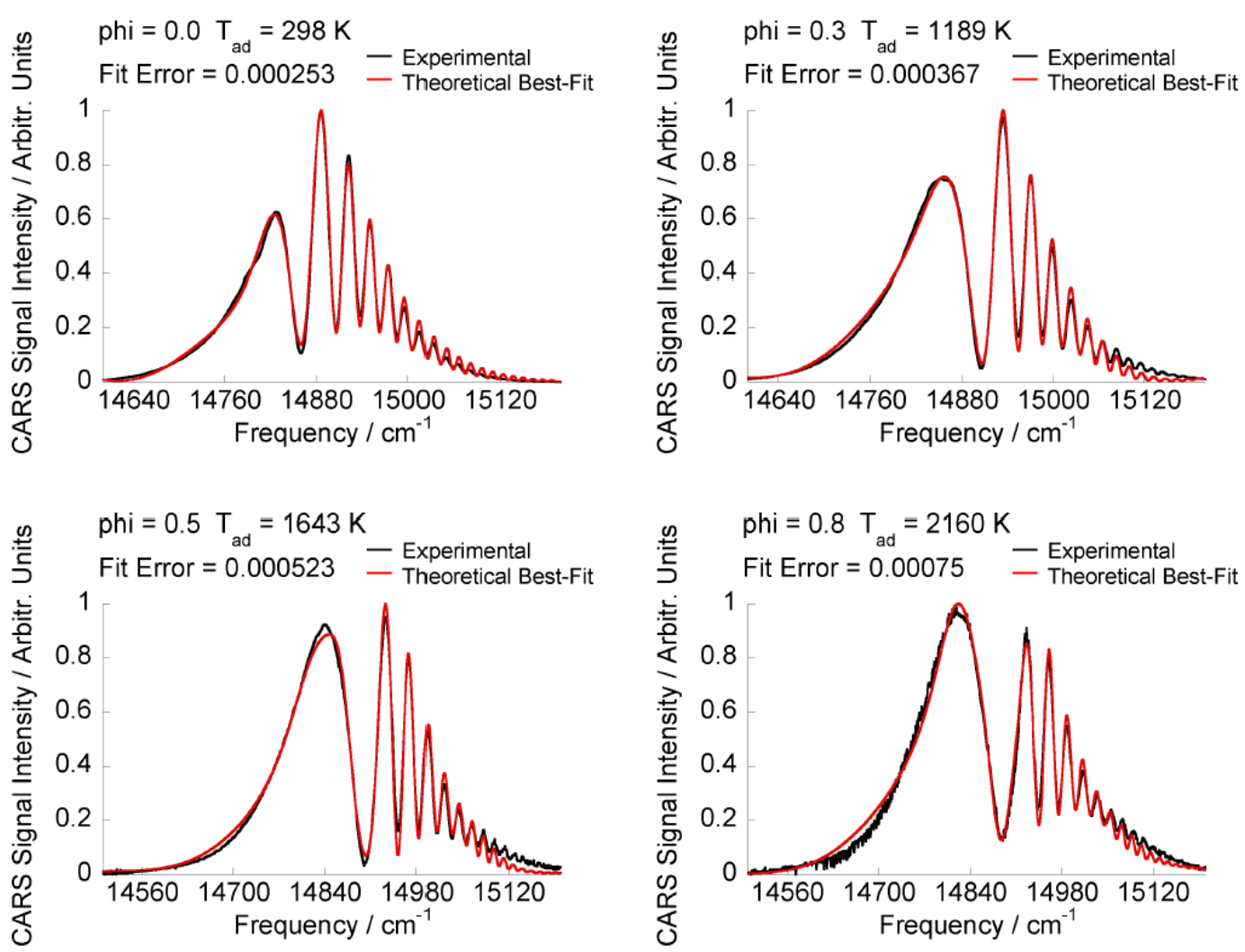

Figure 3: Theoretical fits and experimental reference spectra representing an average of 2000 single-laser-shot measurements in the Hencken burner flame.

Measurements in the Hencken burner at known flame temperatures provided the laser parameters required to theoretically model spectra obtained from flames of unknown temperature. Experimentally obtained reference spectra from each equivalence ratio were theoretically fit using a genetic algorithm based spectral fitting code, developed by Richardson et al. and described in detail in References 20 and 21. During this fitting process, the temperature was held constant at the adiabatic flame temperature and the laser parameters required to generate the theoretical spectrum were varied to obtain the best fit to the experimental spectrum. Each set of laser parameters obtained from this procedure were then held fixed for analysis of CPP fs-CARS spectra from the turbulent GTMC flame. During the analysis of CARS spectra from the GTMC, only the temperature, the ratio of the resonant and nonresonant susceptibilities, and a linear scaling factor for intensity were allowed to vary. For each single-shot, CPP fs-CARS 
spectral fitting was performed using all four sets of laser parameters from room air and the reference flames. The temperature was determined by selecting the spectral fit with the lowest error.

The experimental precision and accuracy of the measurements was determined based on the mean and standard deviation, respectively, of probability density functions generated from 2000 singleshot measurements taken at each calibration condition in the Henken burner. The CPP fs-CARS technique has excellent precision with a typical measured standard deviation of 1.5 to $2 \%$ of the mean flame temperature. Generally, the histogram mean values are a few degrees cooler than the calculated adiabatic flame temperature, though still within the estimated accuracy of $3 \%$. The percentage accuracy of the measurement technique improved with increasing flame temperature. For example, during calibration a Hencken burner flame operated at an equivalence ratio of 0.8 yielded the histogram shown in Figure 4. The mean temperature at $2100 \mathrm{~K}$ was $2.7 \%$ below the adiabatic flame temperature of 2160 $\mathrm{K}$ and the standard deviation was $38 \mathrm{~K}$ or $1.8 \%$.

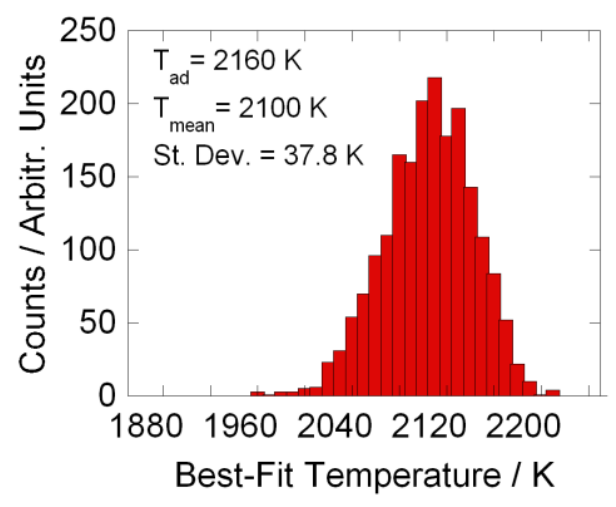

Figure 4: Histogram resulting from 2000 single laser shot temperature measurements in the Hencken burner flame operated at $\varphi=0.8$.

\section{Gas Turbine Model Combustor (GTMC)}

The DLR GTMC used in this work has been the subject of numerous previous studies in which the geometry is described in detail [16,22,23,25], hence only a brief overview is provided here. A 
schematic diagram of the burner is shown in Figure 5. Dry air was supplied to the plenum through an acoustic isolation section with an upstream choked orifice. Fuel and oxidizer flows were controlled by electromechanical mass flow controllers (Porter Series 200). A microphone (Bruel and Kjaer, Type 4939) was installed in the upstream plenum to monitor chamber acoustics. [23] The microphone signal was acquired simultaneously with the laser pulse and the EMCCD gate signals to synchronize the acoustics with the CARS measurements.

The flame studied in this work corresponded to the 'Flame V' condition in the work of Stohr et al. [23]. Air and methane were supplied to the burner at $324 \mathrm{~g} / \mathrm{min}$ and $12.3 \mathrm{~g} / \mathrm{min}$, respectively. This corresponded to a global equivalence ratio of 0.65 and a thermal power of $10 \mathrm{~kW}$. The burner was operated at this condition for thirty minutes, prior to data collection, to allow for thermal stabilization. This operating condition exhibited a thermo-acoustic pulsation at approximately $310 \mathrm{~Hz}$ consistent with previous work.

The time-averaged flow field corresponding to the Flame V condition was measured by Stohr et al. [23] and is shown in Figure 6. The contours are streamlines representing the mean velocity field determined by extensive PIV characterization of the burner and the color scale indicates velocity magnitude in meters per second. The CARS measurement locations (indicated by black squares) were chosen to resolve the spatial structure of the flame, with increased spatial resolution near the burner face. The demonstration of the temporally-resolved CARS temperature measurements in the highly turbulent shear layers near the flame root was of key interest. A precessing vortex core coupled with unsteady flow-flame interactions dominates the flow physics in this region, resulting in highly unsteady temperatures. [16,23,24] 


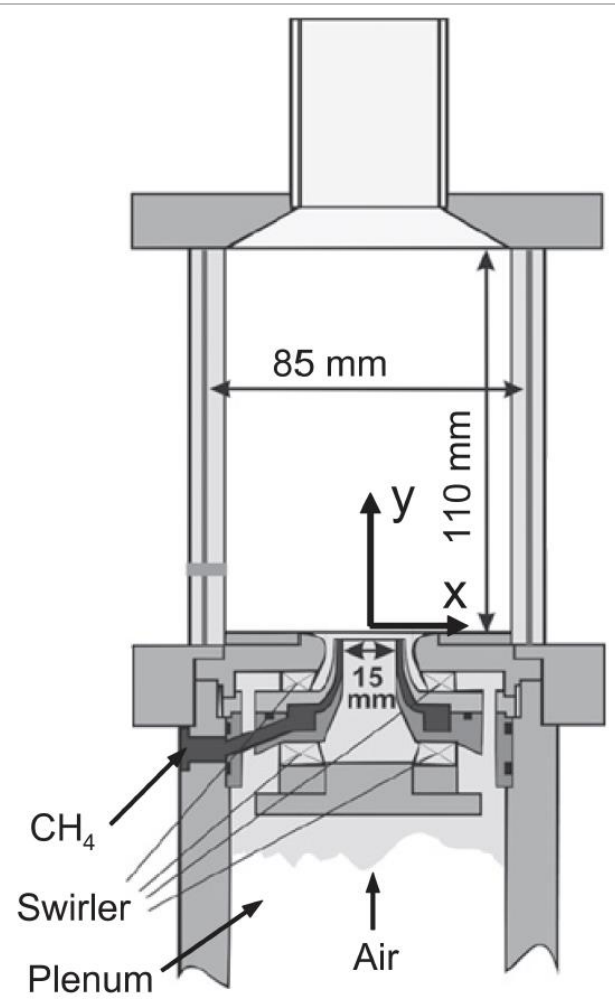

Figure 5: Schematic diagram of DLR Dual-

Swirl GTMC. [23]

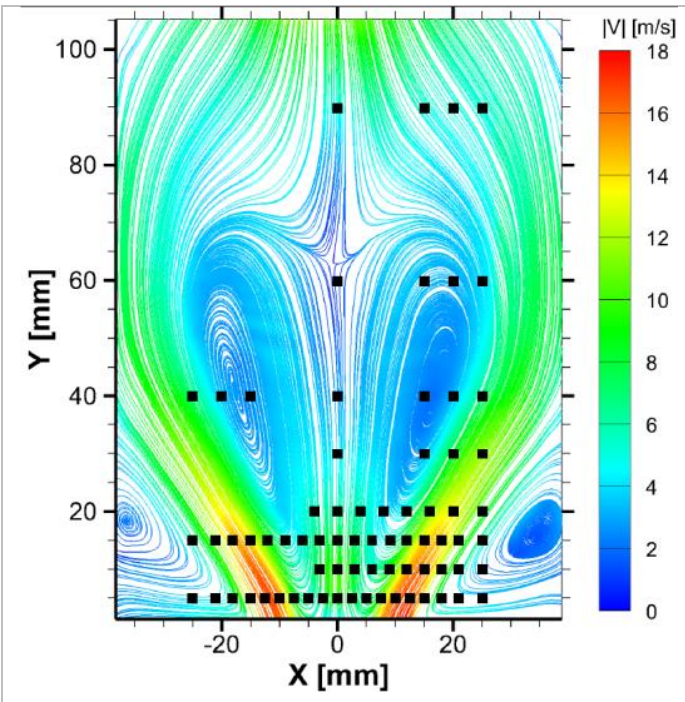

Figure 6: CARS probe-volume locations with respect to mean flow field for the flame 
V operating condition.

\section{Results}

Measurements were performed at the grid locations shown in Figure 6. Analysis of this data is still ongoing, but results from eight locations are shown in Figure 7. For each probe volume location the mean temperature and the standard deviation are reported. The lower limit of temperature for the spectral fitting process was set to $300 \mathrm{~K}$. 

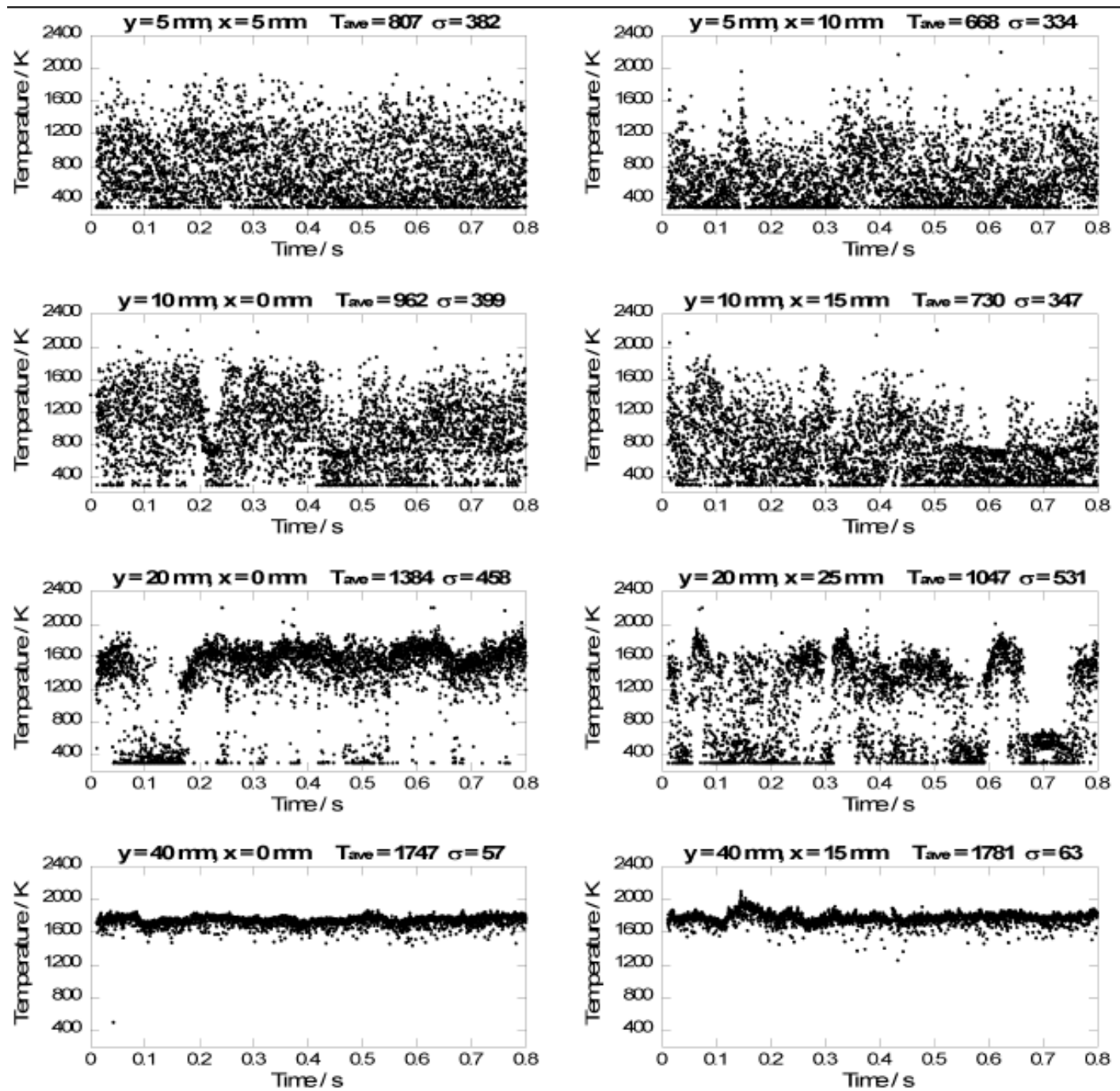

Figure 7: Temperature measurements from 2000 consecutive single-laser-shots for 8 different probe volume locations.

The probe volume locations at $\mathrm{y}=5 \mathrm{~mm}$ and $\mathrm{x}=5$ and $10 \mathrm{~mm}, \mathrm{y}=40 \mathrm{~mm}$ and $\mathrm{x}=15 \mathrm{~mm}$, and along the centerline are located within the inner recirculation zone. In this recirculation zone near the burner exit the measured temperatures vary widely; the variation decreases with axial distance as shown at $\mathrm{y}=20$ and $40 \mathrm{~mm}$ for $\mathrm{x}=0$. Probe volume locations at $\mathrm{y}=10 \mathrm{~mm}$ and $\mathrm{x}=15 \mathrm{~mm}, \mathrm{y}=20 \mathrm{~mm}$ and $\mathrm{x}=$ $25 \mathrm{~mm}$ coincide with the inner shear layer. As expected, the temperature increases with axial distance from the burner exit as combustion products reach equilibrium. The burner operating conditions corresponded to a global adiabatic flame temperature of $1750 \mathrm{~K}$. At the location $\mathrm{y}=40 \mathrm{~mm}, \mathrm{x}=0 \mathrm{~mm}$, the measured mean temperature is around $1747 \mathrm{~K}$, which agrees with the calculated post-flame temperature In order to increase CARS signal levels in the high temperature post flame regions where 
the density is low, the gain of the EMCCD camera was increased. An EMCCD gain setting of 5 was used at axial locations of $\mathrm{y}=15,20$, and $30 \mathrm{~mm}$, and a gain setting of 8 was used for locations at $\mathrm{y}=40$, 60, $90 \mathrm{~mm}$. Without gain, in the high temperature regions, the CARS signal peaks at approximately 70 photoelectrons above background sensor levels. The time history plots for $\mathrm{y}=20 \mathrm{~mm}$, show instances where the temperature measurements appear to bottom out at the $300 \mathrm{~K}$ lower limit. For those laser shots the CARS signal was saturating the detector. We plan to employ a two CCD, two spectrometer system with $10 \%$ of the signal directed to one spectrometer-CCD system and $90 \%$ to the other to overcome this current dynamic range limitation.

To compare with existing thermo-acoustic measurements, eight locations were selected for power spectral density analysis. Results from two of the probe volume locations analyzed are shown in Figures 8 and 9. The plotted data represents the average power spectra of temperature measurements acquired over five separate 5000 laser-shot acquisitions, representing five seconds of data acquisition.

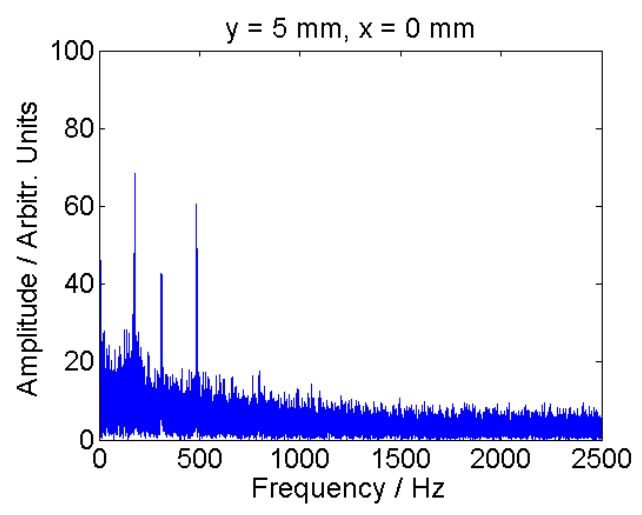

Figure 8: Power spectrum from 25,000 CARS temperature measurements at $y=5 \mathrm{~mm}, x=0 \mathrm{~mm}$. 


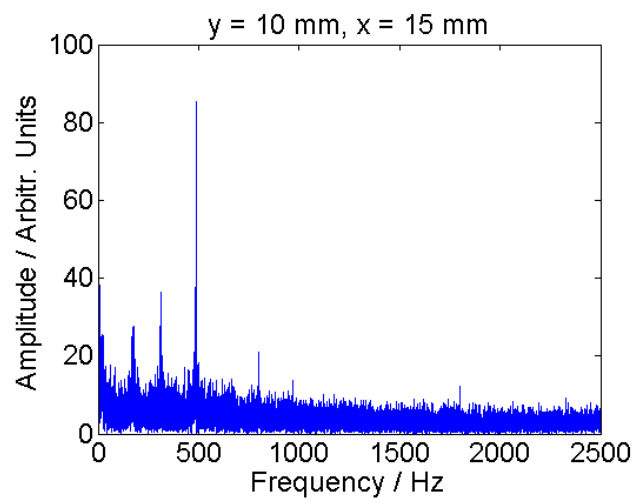

Figure 9: Power spectrum from 25,000 CARS temperature measurements at $y=10 \mathrm{~mm}, \mathrm{x}=15 \mathrm{~mm}$.

The spectra show peaks at $176,308,485$, and $796-805 \mathrm{~Hz}$ for the various axial and radial locations. Boxx et al. also observed a strong peak at $308 \mathrm{~Hz}$, which was determined to be the dominant longitudinal thermo-acoustic pulsation of the flame. [16] The peak at $485 \mathrm{~Hz}$ is likely due to the PVC identified by Stöhr and Meier [22] and observed at $515 \mathrm{~Hz}$ by Boxx. Peaks occurring at 176 and 796$805 \mathrm{~Hz}$ are $\pm 308 \mathrm{~Hz}$ with respect to the PVC frequency and can be attributed to a superposition of thermo-acoustic pulsation and the precessing vortex core. [24] The same phenomenon was documented by Boxx et al. though occurring at 205, 515, and $822 \mathrm{~Hz}$. Here the frequencies are shifted slightly, possibly due to the difference in the ambient operating conditions for each study though similar physics are represented.

\section{Conclusions}

Chirped probe pulse femtosecond CARS has been successfully applied for flame temperature measurements in a model gas turbine combustor with significant swirl and high levels of turbulence. Every laser shot produced some resonant CARS signal; no significant loss of signal due to beam steering, pressure fluctuations, or shear layer density gradients was noticeable. Power spectral density 
analysis of the CPP fs-CARS thermometry results yielded the characteristic thermo-acoustic pulsation frequency previously reported at $308 \mathrm{~Hz}$. These results demonstrate the usefulness of high repetition rate CPP fs-CARS for the study of turbulent combustion.

\section{Acknowledgements}

Funding for this work was provided by the U.S. Department of Energy, Division of Chemical Sciences, Geosciences and Biosciences under Grant No. DE-FG02-03ER15391. The ultrafast laser system was purchased with funding from AFOSR DURIP Grant No. FA9550-09-1-0387.

\section{References}

${ }^{1}$ S. Roy, J. R. Gord, A. K. Patnaik, Prog. Energy Combust. Sci. 36 (2) (2010) 280-306.

${ }^{2}$ S. Roy, P. J. Kinnius, R. P. Lucht, J. R. Gord, Opt Comm. 281 (2) (2008) 319-325.

${ }^{3}$ J. R. Gord, T. R. Meyer, S. Roy, Annu Rev Anal Chem. 1 (1) (2008) 663-687.

${ }^{4}$ T. Lang, M. Motzkus, J. Opt. Soc. Am. B 19 (2) (2002) 340-344.

${ }^{5}$ R. P. Lucht, P. J. Kinnius, S. Roy, J. R. Gord, J. Chem. Phys. 127 (4) (2007) 044316.

${ }^{6}$ R. P. Lucht, S. Roy, T. R. Meyer, J. R. Gord, Appl. Phys. Lett. 89 (25) (2006) 251112.

${ }^{7}$ Richardson, D. R., Lucht, R. P., Roy, S., Kulatilaka, W. D., Gord, J. R., Proc. Combust. Inst. 33 (2011) 839-845.

${ }^{8}$ D. Bangar, C. N. Fineman, R. P. Lucht, 51 ${ }^{\text {st }}$ AIAA Aerospace Sciences Meeting, AIAA 2013-0336, Dallas, TX, 2013.

${ }^{9}$ R. Giezendanner, O. Keck, P. Weigand, W. Meier, U. Meier, W. Stricker, M. Aigner, Combust. Sci. Technol. 175 (4) (2003) 721-741.

${ }^{10}$ R. Giezendanner, P. Weigand, X.R. Duan, W. Meier, U. Meier, M. Aigner, B. Lehmann, J. Eng. Gas Turbul. Power 127 (3) (2005) 492-496.

${ }^{11}$ R. Giezendanner, U. Meier, W. Meier, M. Aigner, Flow Turbul. Combust. 75 (1-4) (2005) 317-333.

${ }^{12}$ R. Giezendanner, U. Meier, W. Meier, J. Heinze, M. Aigner, Appl. Opt. 44 (31) (2005) 6565-6577.

${ }^{13}$ X.R. Duan, W. Meier, P. Weigand, B. Lehmann, Appl. Phys. B 80 (3) (2005) 389-396.

${ }^{14}$ P. Weigand, W. Meier, X.R. Duan, R. Giezendanner-Thoben, U. Meier, Flow Turbul. Combust. 75 (1-4) (2005) $275-292$.

${ }^{15}$ P. Weigand, W. Meier, X.R. Duan, W. Stricker, M. Aigner, Combust. Flame 144 (1-2) (2006) 225-236.

${ }^{16}$ I. Boxx, M. Stöhr, C. Carter, W. Meier, Combust. Flame 157 (8) (2010) 1510-1525.

${ }^{17}$ A. C. Eckbreth, Laser Diagnostics for Combustion Temperature and Species, Abacus Press, UK, 1988.

${ }^{18}$ W. D. Kulatilaka, R. P. Lucht, S. F. Hanna, V. R. Katta, Combust. Flame 137 (4) (2004) 523-537.

${ }^{19}$ A. Satija, R. P. Lucht, Opt Lett. 38 (8) (2013) 1340-1342.

${ }^{20}$ D. R. Richardson, R. P. Lucht, W. D. Kulatilaka, S. Roy, J. R. Gord, Appl. Phys. B 104 (3) (2011) 699-714.

${ }^{21}$ D. R. Richardson, D. Bangar, R. P. Lucht, Opt. Express 20 (9) (2012) 21495-21504.

${ }^{22}$ M. Stöhr, W. Meier, Proc. $3^{\text {rd }}$ European Combustion Meeting, Paper 18-10, Chania, Greece, 2007.

${ }^{23}$ M. Stöhr, I. Boxx, C. D. Carter, W. Meier, Combust. Flame 159 (8) (2012) 2636-2649.

${ }^{24}$ A. M. Steinberg, I. Boxx, M. Stöhr, C. D. Carter, and W. Meier, Combust. Flame 157 (12) (2010) $2250-2266$.

${ }^{25}$ M. Stöhr, R. Sadanandan, and W. Meier, Exp. Fluids 51(4) (2011) 1153-1167. 


\section{Figure Captions}

Figure 1: Schematic diagram of CPP fs-CARS experimental system.

Figure 2: CARS probe volume length measurement.

Figure 3: Theoretical fits and experimental reference spectra representing an average of 2000 single-laser-shot measurements in the Hencken burner flame.

Figure 4: Histogram resulting from 2000 single laser shot temperature measurements in the Hencken burner flame operated at $\varphi=0.8$.

Figure 5: Schematic diagram of DLR Dual-Swirl GTMC. [23]

Figure 6: CARS probe-volume locations with respect to mean flow field for the flame V operating condition.

Figure 7: Temperature measurements from 2000 consecutive single-laser-shots for 8 different probe volume locations.

Figure 8: Power spectrum from 25,000 CARS temperature measurements at $y=5 \mathrm{~mm}, \mathrm{x}=0 \mathrm{~mm}$.

Figure 9: Power spectrum from 25,000 CARS temperature measurements at $\mathrm{y}=10 \mathrm{~mm}, \mathrm{x}=15 \mathrm{~mm}$. 
Ti:Sapphire Oscillator and Regenerative Amplifier
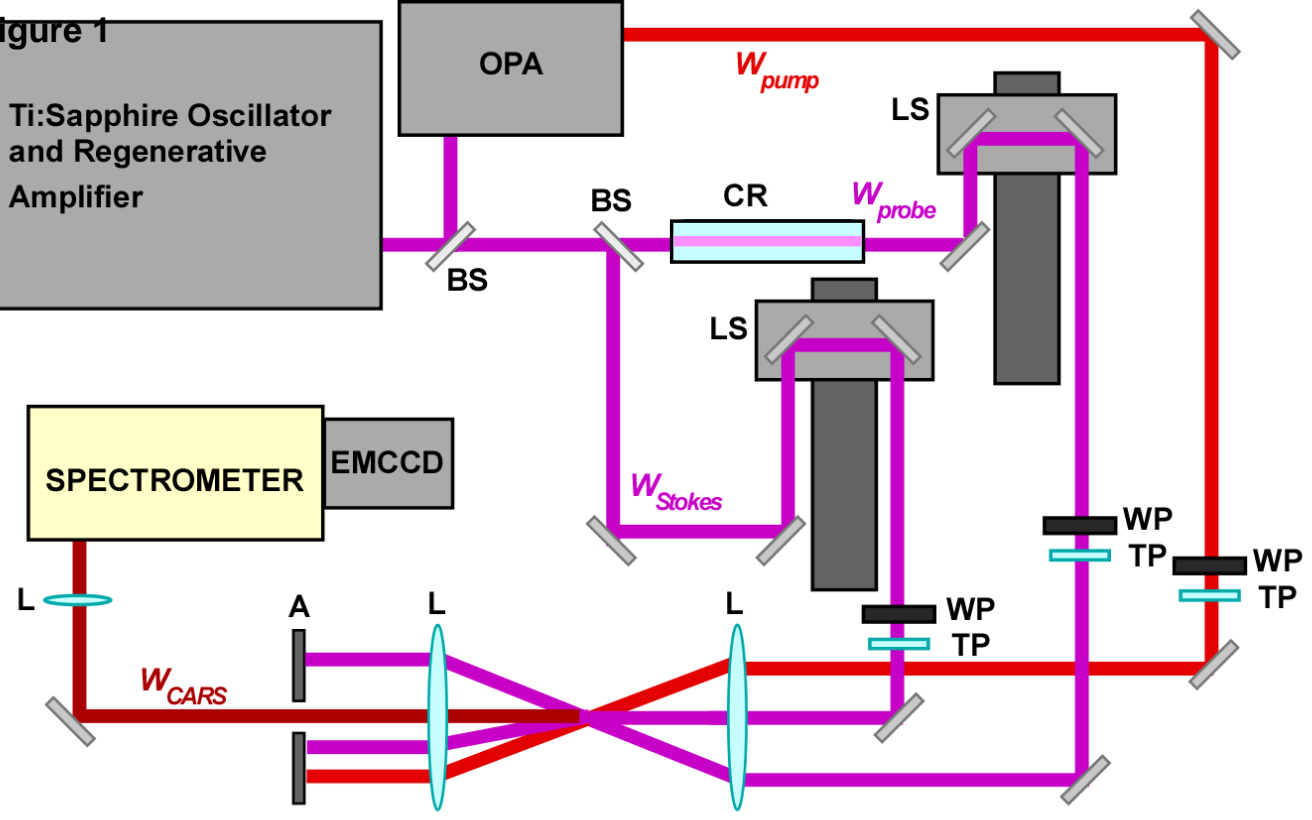

OPA: Optical Parametric Amplifier WP: Zero Order Wave Plate EMCCD: Electron Multiplying Charge-Coupled Device Camera TP: Thin Film Polarizer

BS: Beam Splitter A: Aperture
BS 


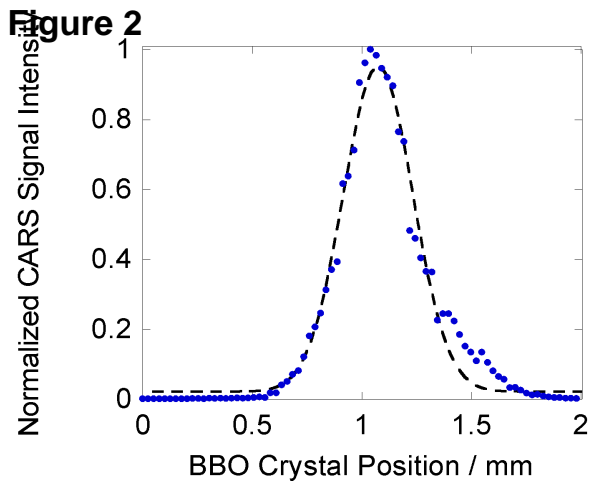




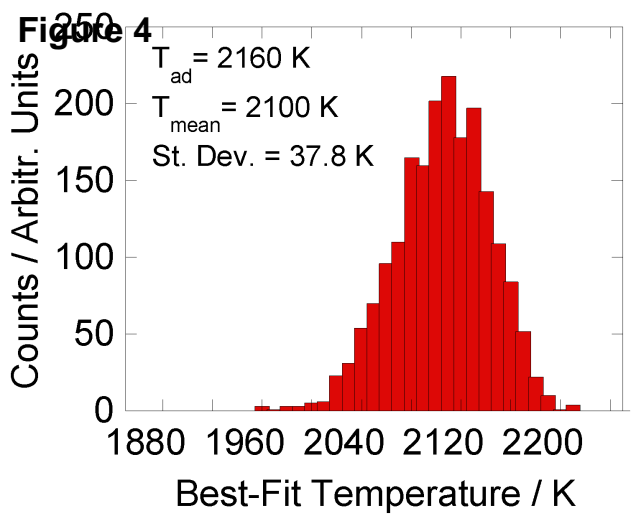




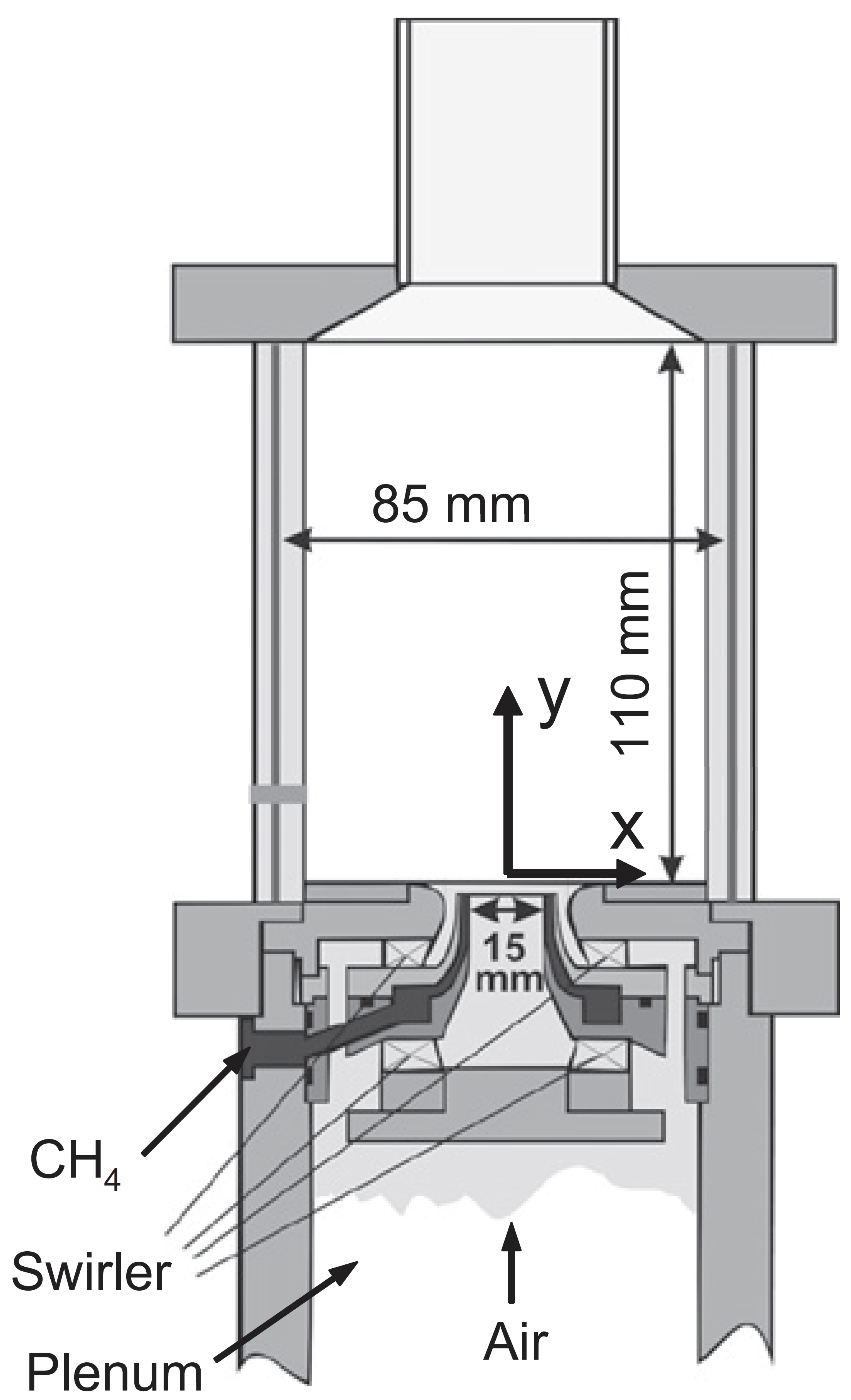




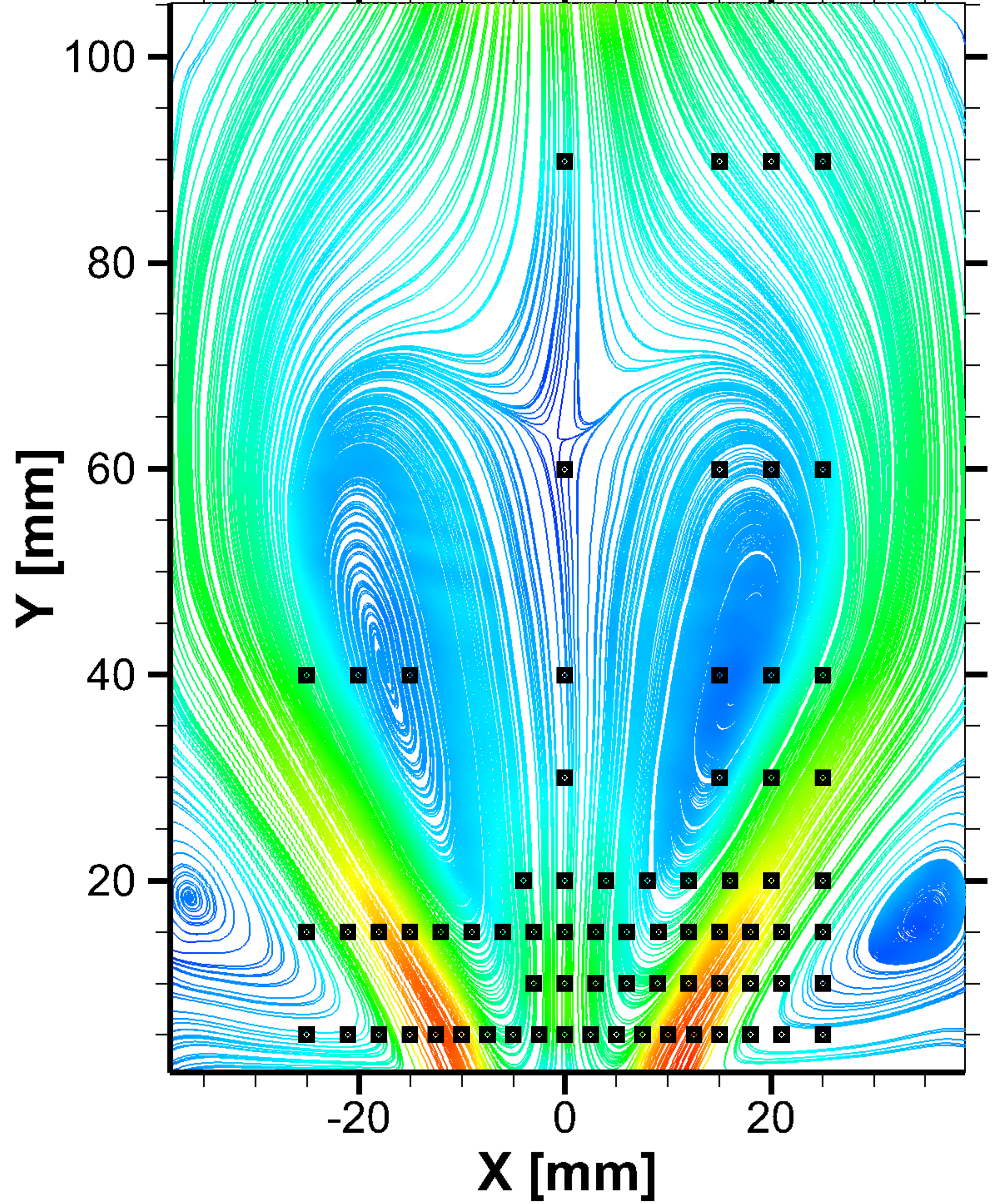

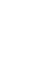

$100+13$
$|\mathrm{V}|[\mathrm{m} / \mathrm{s}]$ 18

16

14

12

10

8

6

4

2

0 


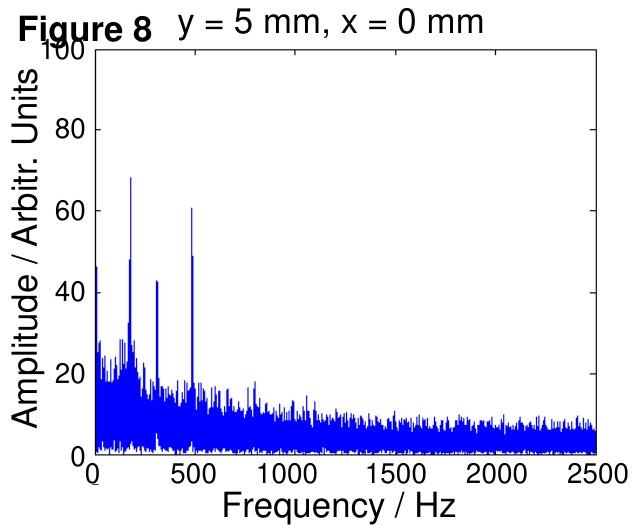




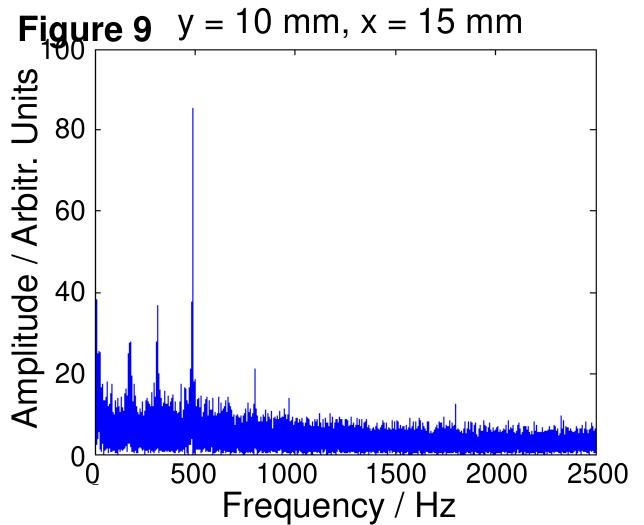

